\title{
A Rare Case of Mural Thrombus in Normal Descending Thoracic Aorta with Literature Review
}

ISSN: 2578-0379

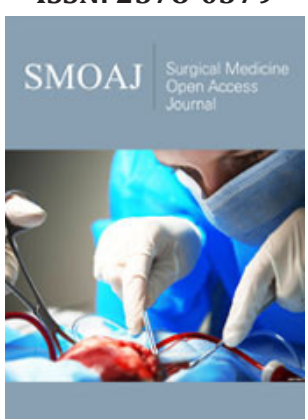

*Corresponding author: Jayesh Sagar, Department of Colorectal \& General Surgery, UK

Submission: 侮 September 17, 2020

Published: 望 September 30, 2020

Volume 3 - Issue 5

How to cite this article: Anil Rai, Shantata J K and Jayesh Sagar. A Rare Case of Mural Thrombus in Normal Descending Thoracic Aorta with Literature Review. Surg Med Open Acc J. 3(5). SMOAJ.000572. 2020 DOI: $10.31031 /$ SMOAJ.2020.03.000572

Copyright@ Jayesh Sagar, This article is distributed under the terms of the Creative Commons Attribution 4.0 International License, which permits unrestricted use and redistribution provided that the original author and source are credited.
Anil Rai ${ }^{1}$, Shantata J. Kudchadkar ${ }^{1}$, Jayesh Sagar *

${ }^{1}$ Department of Colorectal \& General Surgery, UK

\begin{abstract}
Occurrence of a mural thrombus in a diseased descending thoracic aorta (atherosclerotic or aneurysmal) is a well-known and commonly encountered vascular entity. However, thrombus formation in a normal appearing descending thoracic aorta (NADTA) is rarely reported in literature so far. We present an unusual case report with a brief literature review of an aortic mural thrombus (AMT) in descending thoracic aorta in a young male. He presented at our center in the emergency department with acute onset abdominal pain and underwent a contrast enhanced CT scan that confirmed mural thrombus in NADTA. He was conservatively managed with anticoagulation therapy. He had a family history of venous thromboembolism (VTE) in his brother, who had experienced two episodes of deep vein thrombosis at the age of 40 years, managed with anticoagulation. The true incidence, prevalence and exact etiopathogenesis of AMT is still uncertain. However, certain predisposing factors have been described in literature. Through this case report, we aim to highlight the significance of increased awareness required among the general surgeons and radiologists for early and correct diagnosis of this condition, to prevent the unforeseen complications of distal organ/acute limb ischaemia and its associated morbidity and mortality.
\end{abstract}

Keywords: Aortic Mural Thrombus (AMT);Anticoagulation; Multidisciplinary Meeting (MDT),Transthoracic Echocardiography (TTE);Pulmonary Angiography

\section{Introduction}

The Virchow's triad, published by German physician Rudolf Virchow in 1856, described three broad categories of factors- 1. Hypercoagulability, 2. Haemodynamic changes- stasis/ turbulence in blood flow, 3. Endothelial injury/dysfunction); contributing to thrombosis [1,2]. Definite risk factors such as hyper viscosity, coagulation factor mutation (Factor V, Factor II G2021A) or deficiency (antithrombin III, protein C or S), familial dysfibrinogenemia, chronic smoking, nephrotic syndrome, drug abuse, severe trauma/burns, cancer, late pregnancy, steroid/contraceptive use; lead to hypercoagulability and thrombus formation [2]. Delayed diagnosis of this can lead to end organ damage and acute limb ischaemia (ALI) secondary to embolization [3]; resulting in amputation in 13-14\% patients while mortality rate stands at $9-12 \%$ [4]. Approximately $80-85 \%$ of all arterial thrombi and emboli originate due to disturbances in cardiac functions such as atrial fibrillation, valvular abnormalities, prosthetic heart valves, endocarditis, and myocardial infarction. Around $5 \%$ of thrombi arise in diseased aorta, related to either atherosclerosis, dissection or aneurysm. The aortic isthmus is more prone to trauma as compared to the rest of the aorta and hence, is the commonest site of thrombus formation $[3,5,6]$. Incidences of mural thrombus in a NADTA with no associated significant medical history have been rare.

\section{Case Report}

A 37-year- old male presented in the accident and emergency department with a 1-hour history of sudden onset, constant right sided upper abdominal pain of stabbing nature, associated with few episodes of vomiting, but there was no history of any hematemesis or melena. Apart from being chronic smoker (15 pack years) and IV drug abuser, he was otherwise fit and healthy with no other associated symptoms or medical problems. On examination, his abdomen was tender in right upper quadrant with voluntary guarding, but no rigidity. Laboratory investigations revealed white blood cell count-16.2, C-reactive protein-24.5, Creatinine-112. Coagulation screen was deranged with prothrombin time (PT)-11.0, activated 
partial thromboplastin time (APTT)-23.8, International Normalized Ratio (INR)- 0.9. Lactate was 3.4 on venous blood gas analysis. ECG showed normal sinus rhythm. Both chest \& abdominal x-rays were unremarkable. A provisional diagnosis of acute cholecystitis was made, and he was started on IV fluids, antibiotics, and analgesics.

Few hours following admission, he had three episodes of coffee ground vomiting. An esophago- gastro duodenoscopy (OGD) was performed, which revealed few antral erosions, mild duodenitis and esophagitis secondary to hiatus hernia, but no evidence of active bleeding was noted. Subsequently, he underwent a Computed Tomography (CT) of thorax, abdomen, and pelvis with contrast, which eventually showed a large eccentric intraluminal thrombus within the mid- descending thoracic aorta, measuring $5 \mathrm{~cm}$ craniocaudally, which involved the right renal artery. This caused multifocal extensive infarction of the right kidney with little preserved perfused right renal parenchyma. A wedge -shaped area of infarct was also noted within the right lower lung, as shown in (Figure 1-3). The vascular surgery team reviewed the CT images and advised to start the treatment dose of Tinzaparin (11,000 Units). Likewise, thrombosis screen including lupus anticoagulant (LA), JAK-2 mutation study and paroxysmal nocturnal hemoglobinuria (PNH) screen was also investigated.

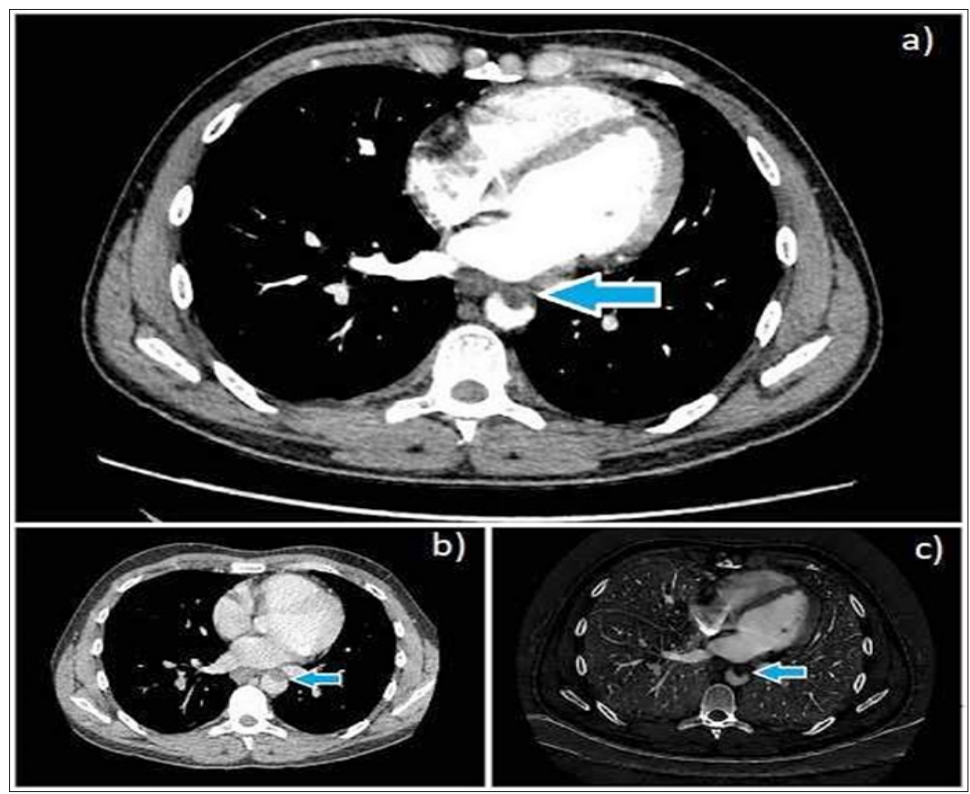

Figure 1: a), b), c): Contrast-enhanced CT images (axial) showing a large eccentric intraluminal thrombus within the mid-descending thoracic aorta (blue arrow).

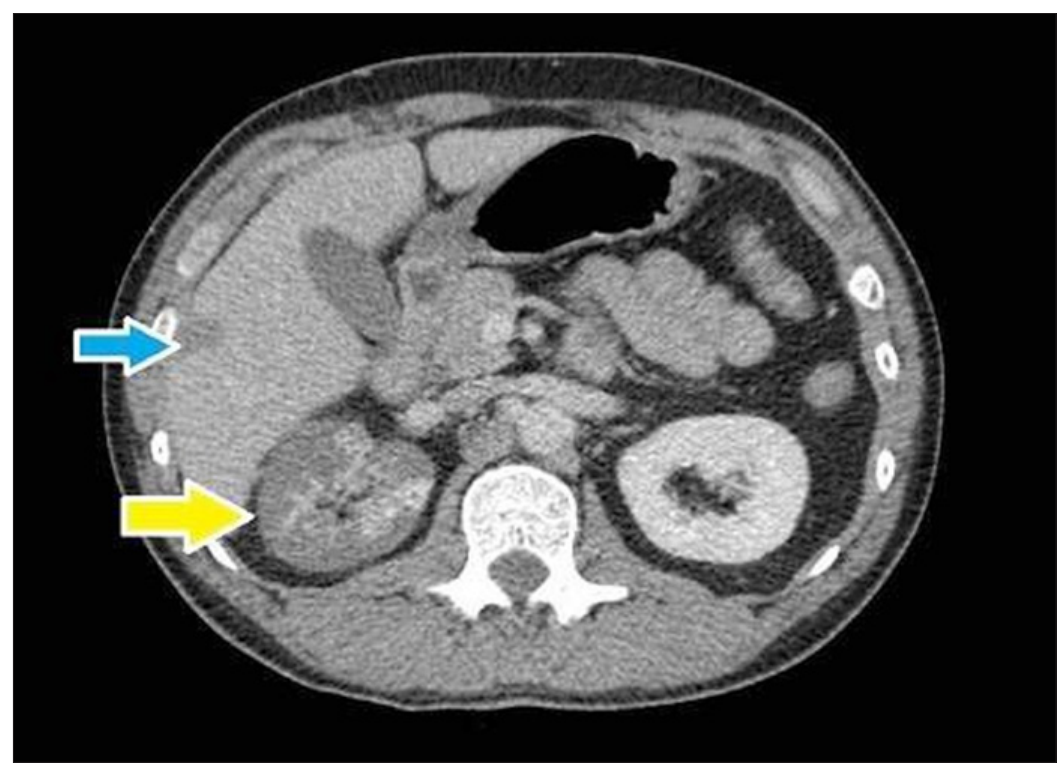

Figure 2: Contrast-enhanced CT images (axial) showing involvement of right renal artery with multifocal extensive infarction of right kidney (yellow arrow) and small infarct in hepatic parenchyma (blue arrow). 


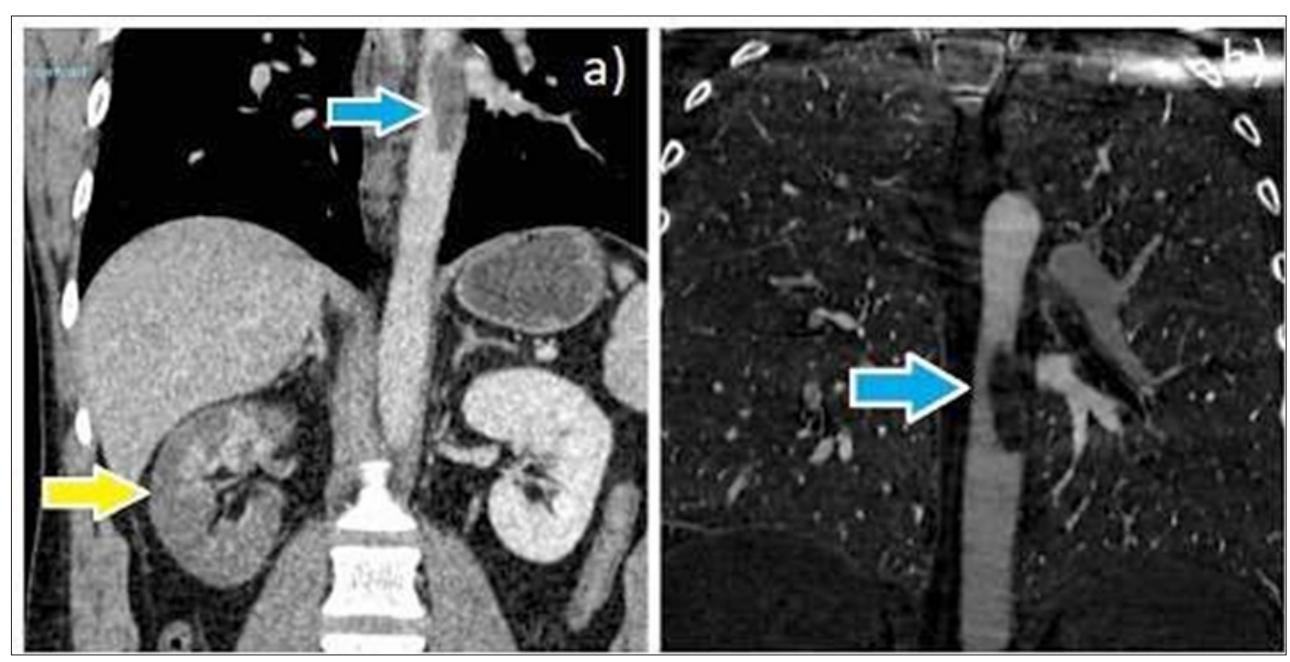

Figure 3: a), b): Contrast-enhanced CT images (coronal) depicting an eccentric intraluminal thrombus within the mid-descending thoracic aorta, measuring $5 \mathrm{~cm}$ craniocaudally (blue arrow) and reduced right renal perfusion (yellow arrow).

Besides, a transthoracic echocardiography (TTE) revealed normal size aortic arch and descending aorta with good forward flow, mild aortic regurgitation and raised pulmonary vascular resistance-attributed to chronic smoking. A week later, CT pulmonary angiography (CTPA) confirmed stable appearance of mural thrombus, small right sided pleural effusion, and few indefinite pulmonary nodules in the right lung. CT Liver triple phase showed no evidence of hepatic infarct, with mild improvement in perfusion of right kidney, along with some excretion into the collecting system on delayed scans. Further tests revealed low complement C3 (0.77), antithrombin-III antigen (59), protein C (57) levels and markedly raised IgG (57.6) levels. This suggested that he was more prone to thrombus formation with possible autoimmune etiology. However, rest of the markers (C4, IgM, ANCA MPO, PR3, lupus anticoagulant, B2 glycoprotein-1 antibodies, protein S) were normal. Also, Factor V Leiden and prothrombin mutation 20210A were not detected. This confirmed no definitive etiology in him. He was started on conservative management with the treatment dose of tinzaparin along with oral warfarin, as advised by the hematology and vascular surgery experts, following his case discussion in MDT. A regular monitoring of INR with an aim to maintain it at 2.5-3.5 was suggested. MDT Vascular Surgery plan was to avoid thoracic aorta stenting in him due to the young age of presentation. He is currently doing well on oral warfarin, awaiting a review in anticoagulation clinic and a repeat CT thorax/ CT angiogram of whole aorta in 3 months' time, to evaluate the thrombus size and rule out dissection/ aneurysm.

\section{Discussion}

Formation of a mural thrombus in normal appearing descending thoracic aorta (NADTA) is an uncommon condition. According to Virchow's triad three factors contribute to the cascade of events leading to thrombus formation and propagation, which ultimately occludes the vessel and produces embolic complications. Thrombi are classified into 3 major groups (white, red and mixed thrombi) depending on the relative number of platelets and RBC's. Occasionally, fresh thrombi have a cystic appearance due to varying degrees of maturity of the clot and results in acoustic boundaries demarcating relatively fresh and more organized areas [7]. AMT as the cause of distal embolization was first described by Williams et al. [8] in 1981 [8]. In his study, all 20 patients with acute embolic occlusion of extremity arteries secondary to unknown etiology underwent aortic thrombectomy.

Further studies by Reber et al. [5], Gagliardi et al. [9], and Machleder et al. [10] also demonstrated presence of mural thrombus in normal aorta devoid of intimal disease. This condition has been described in the literature as spontaneous aortic thrombus, non-occlusive aortic thrombus or primary aortic thrombus [11]. Majority of the arterial emboli originate due to atherosclerosis and pre-existing cardiac disease, accounting for approximately $85 \%$ of limb, visceral or cerebral thromboembolic events [12]. Unexpected aortic mural thrombi (AMI) is recognized as one of the rare causes, whose incidence of detection has increased due to the current advancement in imaging technology [12,13]. Few case reports demonstrate that genetic predisposition might have a role in increasing individual's susceptibility for thrombus formation in normal aorta [14]. Recent studies have shown a correlation between various risk factors and thrombus formation in a blood vessel including hyperviscosity, antiphospholipid syndrome, essential thrombocytopenia, hyperhomocysteinaemia, coagulation factor mutation (Factor V, Factor II G2021A) or deficiency (antithrombin III, protein $\mathrm{C}$ or $\mathrm{S}$ ), familial dysfibrinogenemia, chronic smoking, nephrotic syndrome, drug abuse, severe trauma/burns, cancer, late pregnancy, steroid use and hormone replacement therapy [8]. Prolonged smoking impairs endothelial vasculature, augments 
blood viscosity, has an impact on fibrin network morphology and eventually, leads to either atherosclerotic plaque or blood vessel dilatation $[15,16]$.

A detailed clinical assessment along with radiological assistance helps in making the diagnosis of a mural thrombus. Every patient with thrombosis in normal sized aorta should undergo a thorough evaluation by means of genetic testing and thrombosis work-up, in order to identify the etiology and formulate a definitive treatment. In our case, we have looked at all the predisposing factors and filtered out a few - including chronic smoking, low complement C3 (0.77), antithrombin-III antigen (59), protein C (57) levels, markedly raised IgG (57.6) levels along with family background of VTE; which we believe might have increased his susceptibility to thrombotic activity. Management of a mural thrombus in NADTA has always been challenging. It usually depends on multiple factors classified into- 1 . Patient related such as general condition, preexisting co- morbidities, presence of high-risk factors, severeity of symptoms; 2 . Thrombus related such as location, morphology (sessile/pedicled), nature (stable/floating), underlying pathology, the end organ at risk, urgency of the situation and hence, needs to be individualized [3]. Presence of a floating thrombus in the thoracic aorta is associated with a high risk of peripheral embolism [5]. A complete work-up for hypercoagulability and accurate clinical assessment by an experienced vascular surgeon and haematologist is imperative prior to initiation of the treatment. A search for the other possible causes responsible for the initiation of thrombosis, such as hyperhomocysteinaemia, polycythaemia rubra vera, autoimmune disorders and malignant neoplasm; should be made in addition to the coagulation defects.

Medical treatment mainly involves systemic anticoagulation with heparin and coumarin derivatives in order to maintain an adequate INR. As our patient was young, vascular and haematology specialists at our center recommended therapeutic anticoagulation as the first line therapy. The optimal duration and intensity of anticoagulation therapy, appropriate for the treatment of AMT is not fully known [3]. On the basis of venous literature, Bowdish et al. [11] in their research recommended continuation of therapeutic levels of intravenous heparin or low molecular weight heparin for a minimum of 5 days or till no further evidence of peripheral embolization remains and beyond that, until an adequate intensity of warfarin (INR of 2.5 to 3.5) has been established for 24 to 36 hours [17]. In individuals managed conservatively, further progress of thrombus is monitored with the use of currently available advanced imaging techniques (CTPA, CT angiogram of aorta) [18].

Early initiation of anticoagulation alone is associated with high rates of thrombus resolution and a low incidence rate of reembolization. Surgical/ interventional therapy is indicated in those with mobile thrombus, persistence of thrombus/ re-embolization despite adequate anticoagulation, recurrent embolic episodes, embolic complications and contraindications to anticoagulation. Varied surgical approaches has been described such as thrombolysis, thrombectomy, endovascular repair, segmental aortic resection with reconstruction using prosthetic grafts, endoluminal stent grafts, surgical bypass. More recently, there has been a change in the treatment trend of aortic mural thrombus from surgical to conservative medical management. Although multiple treatment options are available, there are no expert approved guidelines for the same [19]. While some studies suggest aggressive approach to prevent recurrence and risk of peripheral embolization, others prefer a less invasive conservative approach [20]. Many questions still remain unanswered regarding the drug of choice, treatment dose, duration, chances of thrombus recurrence following any form of therapy, dose to prevent future recurrence, therapeutic range of INR to aim for, while on anticoagulation. More evidence in terms of RCT's and meta-analysis is required to form a standardized agreed consensus. Anticoagulation remains as an effective means for controlling and preventing further progression of mural thrombi with longer duration in some patients till the cause is identified and rectified. We suggest having a standardized investigation pathway for similar patients and a dedicated registry of these patients, in order to understand the pathophysiology and formulate a better management plan.

\section{Conclusion}

The isolated development of an aortic mural thrombus (AMT) in an otherwise normal aorta is an infrequent phenomenon. Prompt recognition of this entity and ascertaining its underlying etiopathogenesis is crucial, as it can change the clinical outcomes to a greater extent. All possible etiologies such as infectious, inflammatory, malignant, hypercoagulable, autoimmune and familial aortic ailments must be excluded. It is prudent to perform a complete clinical examination along with a meticulous thrombosis workup, prior to commencing any definitive treatment plan. Lack of current evidence on therapeutic avenues makes the decisionmaking challenging. A discussion with the expert team consisting of vascular surgeons, cardiologists, radiologists, specialty nurses; aids in addressing such difficult cases. Management has to be patient \& thrombus specific. Life- long therapeutic anticoagulation is recommended in majority of cases, unless contraindicated. Through this case report, our primary objective is to create awareness among the general surgeons and radiologists about AMT, in order to avoid its misdiagnosis, delay in recommended therapy and related consequences.

\section{References}

1. Bagot CN, Arya R (2008) Virchow and his triad: A question of attribution. Br J Haematol 143(2): 180-190.

2. Virchow R (1856) Thrombosis and embolism. Vascular Inflammation and Septic Infection. Collected treatises on scientific medicine (in German). Frankfurt am Main: Meidinger \& Sohn. pp. 219-732.

3. Tsilimparis N, Hanack U, Pisimisis G, Yousefi S, Wintzer C, et al. (2011) Thrombus in the non-aneurysmal, non-atherosclerotic descending thoracic aorta-an unusual source of arterial embolism. Eur J Vasc Endovasc Surg 41(4): 450-457. 
4. Eliason JL, Wainess RM, Proctor MC, Dimick JB, Cowan JA, et al. (2003) A national and single institutional experience in the contemporary treatment of acute lower extremity ischaemia. Ann Surg 238: 382-389.

5. Reber PU, Patel AG, Stauffer E, Muller MF, Do DD, et al. (1999) Mural aortic thrombi: An important cause of peripheral embolization. J Vasc Surg 30(6): 1084-1089.

6. Connell JB, Baldrich WJQ (2009) Proper evaluation and management of acute embolic versus thrombotic limb ischemia. Semin Vasc Surg 22(1): 10-16.

7. Davinder PS, Hajira B, Ahmad M, Kunal M (2020) Mural thrombi. In: StatPearls, Stat Pearls Publishing, UK.

8. Williams GM, Harrington D, Burdick J, White RI (1981) Mural thrombus of the aorta: An important, frequently neglected cause of large peripheral emboli. Ann Surg 194(6): 737-744.

9. Gagliardi JM, Batt M, Khodja RH, Le bas P (1988) Mural thrombus of the aorta. Ann Vasc Surg 2: 201-204.

10. Machleder HI, Takiff H, Lois JF, Holburt E (1986) Aortic mural thrombus: An occult source of arterial thromboembolism. J Vasc Surg 4: 473-478.

11. Bowdish ME, Weaver FA, Liebman HA, Rowe VL, Hood DB, et al. (2002) Anticoagulation is an effective treatment for aortic mural thrombi. J Vasc Surg 36(4): 713-719.

12. Abbott W, Maloney R, McCabe C, Lee C, Wirthlin LS, et al. (1982) Arterial embolism: A 44-year perspective. Am J Surg 143(4): 460-464.

13. Lau LS, Blanchard DG, Hye RJ (1997) Diagnosis and management of patients with peripheral macro emboli from thoracic aortic pathology. Ann Vasc Surg 11(4): 348-353.

14. Beldi G, Bissat A, Eugster T, Gurke L, Stierli P, et al. (2002) Mural thrombus of the aorta in association with homozygous plasminogen activator inhibitor type 1 (PAI-1)-675(4G) and heterozygous GP Ia 807C/T genotypes. J Vasc Surg 36: 632-634.

15. Kiowski W, Linder L, Stoschitzky K, Pfisterer M, Burckhardt D, et al. (1994) Diminished vascular response to inhibition of endotheliumderived nitric oxide and enhanced vasoconstriction to exogenously administered endothelin-1 in clinically healthy smokers. Circulation $90(1): 27-34$

16. Pretorius E, Oberholzer HM, Spuy WJ, Meiring JH (2010) Smoking and coagulation: The sticky fibrin phenomenon. Ultrastruct Pathol 34(4): 236-239.

17. Choukroun EM, Labrousse LM, Madonna FP, Deville C (2002) Mobile thrombus of the thoracic aorta: Diagnosis and treatment in 9 cases. Ann Vasc Surg 16(6): 714-722.

18. Habib H, Judy H, Patricia JW, Joseph D (2013) Mural thrombus in the normal-appearing descending thoracic aorta of a chronic smoker. Tex Heart Inst J 40(5): 619-622.

19. Meyermann K, Trani J, Caputo FJ, Lombardi JV (2017) Descending thoracic aortic mural thrombus presentation and treatment strategies. Journal of Vascular Surgery 66(3): 931- 936.

20. Ramy M, Robert G, Ashish C, Julian JB, Elvis C, et al. (2019) Peripherally embolizing aortic thrombus: The work-up, management, and outcome of primary aortic thrombus. Case Reports in Cardiology. 